\title{
Theme : La Cour de Repression des Infractions Economiques et du Terrorisme (Criet)
}

\author{
Edouard Cyriague Dossa
}

\section{INTRODUCTION}

La République du Bénin est un Etat de l'Afrique de l'Ouest situé entre le Nigéria, le Togo, le Burkina-Faso, le Niger et l'Océan Atlantique. Peuplé d'environs dix millions d'âmes, ce pays fait partie de plusieurs Organisations étatiques autant qu'il est partie à plusieurs conventions internationales.

Etat démocratique depuis vingt-neuf (29) ans, le Bénin se fonde sur la loi fondamentale du 11 décembre 1990 qui consacre la séparation des pouvoirs.

En effet, de cette cartographie démocratique, s'élèvent trois pouvoirs (l'Exécutif, le Législatif et le Judiciaire) appelés, chacun, à accomplir les tâches constitutionnelles qui sont les siens. Régi par les dispositions du Titre VI de la constitution du Bénin, le pouvoir judiciaire est exercé par les Cours et Tribunaux. C'est la Loi n²001-37 du 27 août 2002 qui l'a organisé en Tribunaux d'instance, Cours d'Appel et Cour Suprême avec, depuis l'avènement de l'Organisation pour l'Harmonisation en Afrique du Droit des Affaires (OHADA), exigence de faire connaitre les pouvoirs élevés contre les arrêts rendus dans les contentieux d'affaires par la Cour Commune de Justice et d'Arbitrage (CCJA) sise à Abidjan en République de la Côte d'Ivoire.

Au textuel, le Bénin dispose de trente-un (31) tribunaux dont trois de Commerce et de six (06) Cour d'Appel dont trois de Commerce.

De ces juridictions, dix-neuf (19) sont fonctionnelles avec un effectif actuel en Magistrat pour tout le pays de deux cent dix-huit (218) à ce jour.

C'est avec cet arsenal que le pouvoir judiciaire fait face aux litiges de tous ordres dont notamment les infractions traditionnelles, un peu pour désigner le vol, l'abus de confiance, l'escroquerie, les infractions de sang etc...

Cette force de répression, à l'épreuve, s'est révélée impuissante pour plusieurs causes dont les plus élevées sont les suivantes : la corruption, la relative indépendance, la désuétude des textes, l'inconstance de la jurisprudence, le défaut de formation continue des Magistrats, la politisation à outrance du milieu judiciaire mais et surtout l'émergence de nouvelles infractions, pour prendre l'exemple de la cybercriminalité, le blanchiment de capitaux, le terrorisme et son financement, auxquels il faut ajouter toutes les infractions économiques et financières lesquelles de par leur mode de commission viennent des fois remettre en cause les principes sacro-saints du droit pénal (général et spécial) ainsi que le droit processuel lui-même.

Arrivé au pouvoir en 2016, l'actuel Chef de l'Etat, le Président Patrice Athanase Guillaume TALON, affecté par ce diagnostic du secteur de la justice et ayant besoin au 
même moment de sa meilleure forme pour l'accompagner dans l'assainissement économique du pays a considéré ce disfonctionnement comme un frein au développement de la Nation et a souhaité puis obtenu l'actualisation des textes d'organisation, de fond et de procédure du pouvoir judiciaire.

$\mathrm{Si}$, pour des raisons tenant aux considérations politiques, cette volonté est abîmée dans la recherche de la révision de la constitution afin d'instituer une Cour des Comptes comme c'est le cas des Etats de l'UEMOA, elle a tout de même connu une fortune sur le terrain pénal avec la création de la Cour de Répression des Infractions Economiques et du Terrorisme (CRIET) dont l'une des racines inspiratrice est perçue dans les textes fondant la Cour de Répression de 1'Enrichissement Illicite (la CREI) au Sénégal.

Née, à la faveur de la loi n²018-13 du 02 juillet 2018 modifiant et complétant la loi $n$ `2001-37 du 27 août 2002 portant organisation judiciaire en République du Bénin, la Cour de Répression des Infractions Economiques et du Terrorisme (CRIET) dispose d'un personnel Magistrat qui est au nombre de dix-huit (18), lequel est installé le lundi 27 août 2018. Elle a rendu sa première décision à la première audience des Flagrants délits tenue le jeudi 13 septembre de la même année.

Il faut signaler qu'en dix (10) mois d'existence, la CRIET fait l'objet d'une appréciation mitigée. Tandis que les uns y voient un instrument nécessaire au regard des courageuses décisions qu'elle rend, lesquelles disciplinent notoirement les habitudes naguère, criminelles, d'autres, cependant, lui tirent à boulets rouges dessus.

Dans ce cadre, les questionnements ne peuvent manquer de prendre le versant d'une problématique : " Etait-il indispensable de créer la Cour de Répression des Infractions Economiques et du Terrorisme? Dans l'affirmative a-t-elle tous les atouts de l'efficacité attendue d'elle?"

C'est à cette problématique à double branche de préoccupation que nous allons répondre en nous autorisant de discuter de la pertinence de la Cour dans une première partie avant de juger de sa capacité à conférer aux fruits, les promesses des fleurs dans une seconde partie.

\section{I- LA PERTINENCE}

La Cour de Répression des Infractions Economiques et du Terrorisme (CRIET) s'est révélée être une Cour très indispensable à l'Etat béninois. Si les preuves de cette nécessité se trouvent pour l'essentiel dans le contexte de sa création (A), il n'en demeure pas moins évéré qu'elle a des fondements ayant pour appui les critiques ancestrales de la justice (B).

\section{A- Une pertinence contextuelle}

Le contexte dans lequel la CRIET est créée fournit à lui-même, les motifs de l'institution de la Structure. Il concerne les points suivants : 


\section{Une Cour nécessaire :}

La Cour s'inspire timidement des avancées organisationnelles du Niger, du Mali et du Burkina Faso, pays dans lesquels il y a des sections terroristes, d'une part et des sections d'infractions économiques d'autre part, mais avec certitude du cas du Sénégal où est créée la Cour de répression de l'Enrichissement Illicite (CREI). Seulement, au regard de l'assiette élargie de la compétence de la CRIET, on peut affirmer que le Bénin est devenu le modèle de son modèle.

Avec le législateur traditionnel, coutumier des infractions sobrement étudiées dans les universités mais dont la poursuite posait quelques problèmes aussi bien de fond que de procédure, les juridictions de droit commun ne manquaient pas de puiser leurs solutions dans la doctrine et la jurisprudence.

De nos jours, le droit pénal est confronté à la complexité des infractions dites émergentes pour lesquelles, la poursuite des auteurs a besoin de quelques particularités et de quelques spécificités dont l'efficacité requise échappe à la classique manière de mener les enquêtes.

Ces nouvelles formes d'infractions qui défient les règles de procédure ordinaires sont contemporaines et générées par les nouvelles formes de vies dues soit à la prolifération des armes, l'extrémisme violente, l'envie de s'enrichir sans un effort licite.

Relativement au traitement des crimes de terrorisme, des délits ou crimes économiques ainsi que du trafic de stupéfiants et d'autres infractions connexes, la création de cette cour vient à point nommé, pour combler un vide dans une organisation juridique béninoise.

L'existence de la CRIET intervient dans un contexte de réformes économiques et d'une atmosphère d'insécurité dans la sous-région, causée notamment par les actions de groupes terroristes tels que la secte Jama'atuAhlis Sunna Lidda'awatiwal-Jihad (JAS), mieux connue sous le nom de Boko Haram au Nigeria, Al-Qaïda au Maghreb islamique (AQMI), le Mouvement National de Libération de l'Azawad (MNLA), le Mouvement pour l'Unicité et le Jihad en Afrique de l'Ouest (MUJAO) et Ansar Dine au Mali.

\section{Un accompagnement attendu :}

Pour conduire son Programme d'Actions de Gouvernement, le pouvoir en place en vue d'atteindre ses objectifs de bien-être de la population a besoin d'une Justice réellement indépendante, impartiale et dont les décisions sont rendues dans des délais raisonnables;

\section{Une image à corriger}

- Suivant les critiques, pour la plupart fondées, l'image du Bénin, a commencé par être sérieusement écornée au plan international parce qu'il est devenu une plaque tournante de la drogue et un nid criminogène de la cybercriminalité.

- C'est ce qui a amené l'Ambassade des Etats-Unis près le Bénin, à élaborer et édicter un Manuel de procédure afférent au trafic de drogues et autres substances psychotropes. 


\section{Un ministre de la justice précédent éclairé et pragmatique}

Le Bénin avait la chance d'avoir un Ministre de la Justice et de la Législation qui était Président de la Commission des Lois à l'Assemblée Nationale et qui est à la fois, Avocat et Professeur d'Université.

\section{Un ministre de la justice actuel compétent, un bon relai}

Le présent Garde des Sceaux, Ministre la Justice et de la Législation a vite mis en place la Cour et ne cesse de constater qu'elle réalise des avancées satisfaisantes.

\section{Un président de la République déterminé}

Aucune juridiction ne peut prospérer si elle n'a la faveur de la volonté politique. Le président de la République a accepté accompagner la CRIET autant que celle-ci se distinguera par ses résultats objectifs.

\section{B- Une pertinence fondée}

La création de la Cour trouve racine dans des facteurs qui abîment déjà son image. Elle tient sur plusieurs points.

\section{Une compétence en déficit}

- La connaissance et la maîtrise que les magistrats doivent avoir des infractions émergentes étaient devenues douteuses et avaient l'élan commun de l'à peu près

\section{Les constats déterminants :}

- La disparité des décisions de justice : elle s'observe, soit d'une chambre correctionnelle à une autre au sein d'une même juridiction, soit d'une juridiction à une autre, sur le même territoire national et d'une époque à une autre;

- Le manque de célérité dans des dossiers d'envergure. C'est l'exemple constaté dans l'affaire dénommée ICC-Services qui n'est que l'incarnation nationale des affaires de Charles PONZI et de Bernard MADOFF. La lenteur serait dû au fait que les auteurs de cette vague d'escroquerie pour laquelle les auteurs sont poursuivis aurait impliqué des Hommes politiques de l'époque et au plus haut sommet.

- L'impunité de bien des auteurs : l'exemple de l'ex Greffier en Chef du Tribunal de Première Instance de Cotonou, Séidou ABOU qui a détourné une somme de plus deux milliards (2.000.000.000) FCFA au Greffe dudit Tribunal rendant aussi bien les justiciables que l'Etat béninois, victimes.

- Une corruption ambiante du milieu judiciaire non rassurante; 
- Il est à retenir que, cependant, la CRIET offre des garanties suffisantes au niveau de la qualité de sa composition. En effet,

- La présidence de la Cour est tenue par un Président permanent nommé à la suite d'une enquête de moralité sensée lui être favorable et compte tenu de ses connaissances des matières entrant dans la compétence surtout matérielle de l'Institution. Il est accompagné, dans ses offices par quatre magistrats accesseurs et deux magistrats suppléants.

- Le Parquet spécial : Il est dirigé par un Procureur spécial également permanent nommé dans les mêmes conditions.

- La Commission d'instruction. Elle est présidée par un Magistrat qui la dirige avec quatre juges accesseurs.

- La Chambre des Liberté et de la Détention : Elle est dirigée par une Présidente et deux assesseurs, tous magistrats.

\section{II- L'EFFICACITE}

La Cour de Répression des Infractions Economiques et du Terrorisme s'est très tôt voulue être une Cour efficace; en tout cas, ce sont ses ambitions. Cette force de conviction qu'elle veut imprimer à ses œuvres se laisse déjà prouvée (A) tout comme elle se justifie (B).

\section{A- Une efficacité probante}

Elle se base sur les progrès réalisés, lesquels s'énumèrent comme suit :

- La confusion de rigueur en ce qui concerne la délinquance en col bleu et celle en col blanc.

- La première forme de la délinquance implique les plus souvent les membres des communautés défavorisées souffrant d'une vulnérabilité économique qui se traduit souvent par un ostracisme social. Il s'agit souvent d'agents ne disposant que de leurs mains comme outils de travail, ayant des revenus peu confortables pour subvenir à leurs besoins. Il s'agit en réalité d'une délinquance de survie.

- La seconde forme est synonyme d'une délinquance de confort. Elle est d'une gravité avérée et met souvent à rude épreuve la stabilité socio-économique et touche aux fondamentaux de l'Etat.

- Si face aux deux formes, les juridictions traditionnelles font un traitement différentiel des condamnations en faveur de la première, à la CRIET, elles sont logées à la même enseigne dans la rigueur qui caractérise cette Cour.

- Les infractions qui relèvent de la compétence de la Cour ont connu une chute dans les statistiques en si peu de temps. Il s'agit, notamment du trafic de drogues et autres substances psychotropes, de la cybercriminalité, du détournement des deniers publics, de la corruption et du blanchiment de capitaux;

- Des pratiques socio-administratives ont changé dans le pays; 
- Aucun Agent public ne veut commettre la moindre infraction et qui doit l'amener devant la CRIET;

- Des encouragements et félicitations reçus du pouvoir en place et des Organisations internationales (le Groupe Intergouvernemental pour la lutte contre le Blanchiment d'Argent en Afrique de l'Ouest (GIABA), l'Office des Nations Unies contre la Drogue et le Crime (l'ONUDC) et l'Institut international pour la justice et l'état de droit (IIJ IIJ);

- Une constante invite du Pouvoir en place à poursuivre n'importe qui, même si l'auteur est de la mouvance présidentielle;

- La poursuite et la condamnation à des peines fermes d'emprisonnement de quelques richissimes béninois et du Préfet du Littoral, dans une affaire de gestion domanial ayant coûté à son ministre de tutelle, le ministre de la décentralisation, son poste ministériel;

- La poursuite de quelques barrons de la drogue qui n'étaient pas inquiétés par la justice béninoise, prise sous sa forme traditionnelle.

\section{B- Une efficacité fondée}

Les progrès réalisés par la CRIET ont plusieurs causes.

- La qualité des magistrats qui animent la cour : Ils sont intègres, diligents et honnêtes; bref, ils bénéficient, sauf preuve contraire, de la présomption des qualités attendus d'un prototype de Magistrat; Ce qui rassure les justiciables.

- Ils ont, pour les deux Magistrats qui y sont permanents (Le Président de la Cour et le Procureur Spécial) une avance de connaissances sur les infractions émergentes qui relèvent de la compétence de la Cour;

- Le déferrement est fait dans les conditions d'audience;

- Les peines privatives de liberté infligées aux auteurs avérés sont réellement fortes et celles patrimoniales, presque systématiques.

\section{CONCLUSION}

Elle va concerner les insuffisances et les risques liés à la fonction.

Les insuffisances : on en a de plusieurs ordres :

Au plan textuel : La loi 2018-13 portant création de la CRIET est souvent critiqué sur son manque d'organisation du double degré de juridiction.

Au plan matériel : l'immeuble qui abrite la Cour n'est pas adapté pour les besoins d'une juridiction, ce qui fait que l'accommodation n'a pas encore réussi;

Au plan sécuritaire : La CRIET a déjà fait l'objet d'attaque au mois de mai 2019 où, de nuit profonde, des assaillants ont tenté de l'incendier.

Une forte probabilité de contrariétés :

La contrariété viendra éventuellement de l'opposition pouvant exister entre deux décisions rendues par la Cour de Répression des Infractions Economiques et du Terrorisme et la Haute Cour de Justice. 
- La première contradiction peut provenir de l'appréciation des faits par chacune de ces deux instances judiciaires, au regard de son domaine de compétence. Il peut, en effet donc arriver que l'une d'elles, dans l'appréciation des faits, aboutisse à un non-lieu, une relaxe ou acquittement, selon le cas de figure alors que la seconde entre en condamnation au regard des même faits.

- La seconde forme de contrariété va se jouer sur le terrain des peines de condamnation. L'une peut être sévère dans sa condamnation alors que la seconde peut être souple devant la même procédure qui n'est différente qu'au regard des justiciables, toute chose étant égale par ailleurs.

Pour éviter ce risque probable, voire même évident, la seconde juridiction qui prend son dossier peut chercher à aligner sa condamnation sur celle de la première, perdant ainsi son indépendance. 